\title{
Direct approach to the problem of strong local minima in calculus of variations
}

\author{
Yury Grabovsky • Tadele Mengesha
}

Received: 20 September 2007 / Accepted: 22 October 2007 / Published online: 14 February 2008

(C) Springer-Verlag 2008

\section{Erratum to: Calc. Var. (2007) 29:59-83 \\ DOI 10.1007/s00526-006-0056-7}

The proof of Theorem 5 does not prove the statement in Theorem 5. The problem is that the cut-off functions $\theta_{k}^{r}(\boldsymbol{x})$ that vanish on $\partial \Omega$ cannot correctly recover the function $\mathcal{I}\left(\boldsymbol{x}_{0}, \boldsymbol{x}_{0}\right)$ at the points $\boldsymbol{x}_{0} \in \partial \Omega_{1}$, where the measure $\tilde{\pi}$ has a non-zero mass. This problem is easily fixed by using cut-off functions $\theta_{k}^{r} \in C_{0}^{\infty}\left(B\left(x_{0}, r\right)\right)$ that do not vanish on $\partial \Omega$, if $x_{0} \in \partial \Omega$. We require that $\theta_{k}^{r}(\boldsymbol{x}) \rightarrow \chi_{B\left(x_{0}, r\right)}(\boldsymbol{x})$, as $k \rightarrow \infty$ for all $\boldsymbol{x} \in \mathbb{R}^{d}$. This change corrects the Proof of Theorem 5 but makes the arguments in Step 4 in the Proof of Theorem 2 (p. 82) invalid, since $\theta_{k}^{r}(\boldsymbol{x}) \boldsymbol{v}_{n}(\boldsymbol{x})$ no longer has to vanish on $\partial B_{\Omega}\left(\boldsymbol{x}_{0}, r\right) \cap \partial \Omega$. This problem is fixed by augmenting the statement of the Decomposition Lemma (Lemma 1). We claim that it is possible to modify sequences $z_{n}$ and $\boldsymbol{v}_{n}$ is such a way that they vanish on $\partial \Omega_{1}$, while satisfying all the other properties required by Lemma 1 . Using the modified functions in the consequent analysis leads now to the functions $\theta_{k}^{r}(\boldsymbol{x}) \boldsymbol{v}_{n}(\boldsymbol{x})$ that do vanish on $\partial B_{\Omega}\left(\boldsymbol{x}_{0}, r\right)$.

Theorem 1 Suppose the sequence of functions $\psi_{n} \in W^{1, \infty}\left(\Omega ; \mathbb{R}^{m}\right)$ that vanish on $\partial \Omega_{1}$ (relatively open subset of a $C^{1}$ boundary $\partial \Omega$ of $\Omega$ ) is bounded in $W^{1,2}\left(\Omega ; \mathbb{R}^{m}\right)$. Suppose $\alpha_{n} \rightarrow 0$, as $n \rightarrow \infty$ is a sequence of positive numbers such that $\alpha_{n} \boldsymbol{\psi}_{n} \rightarrow \mathbf{0}$ in $W^{1, \infty}$ weak-*. Suppose $z_{n}$ and $\boldsymbol{v}_{n}$ are as in Lemma 1, i.e.

(a) $\boldsymbol{\psi}_{n}(\boldsymbol{x})=\boldsymbol{z}_{n}(\boldsymbol{x})+\boldsymbol{v}_{n}(\boldsymbol{x})$

(b) For all $\boldsymbol{x} \in \Omega \backslash R_{n}$ we have $z_{n}(\boldsymbol{x})=\boldsymbol{\psi}_{n}(\boldsymbol{x})$ and $\nabla \boldsymbol{z}_{n}(\boldsymbol{x})=\nabla \boldsymbol{\psi}_{n}(\boldsymbol{x})$;

(c) The sequence $\left\{\left|\nabla z_{n}\right|^{2}\right\}$ is equiintegrable;

The online version of the original article can be found under doi:10.1007/s00526-006-0056-7.

Y. Grabovsky $(\bowtie)$

Temple University, Philadelphia, PA 19122, USA

e-mail: yury@temple.edu

T. Mengesha

Coastal Carolina University, Conway, SC 29528, USA

e-mail: mengesha@coastal.edu 
(d) $\boldsymbol{v}_{n} \rightarrow 0$ weakly in $W^{1,2}\left(\Omega ; \mathbb{R}^{m}\right)$;

(e) $\left|R_{n}\right| \rightarrow 0$ as $n \rightarrow \infty$;

(f) $\alpha_{n} \nabla z_{n}$ is bounded in $L^{\infty}$;

Then there exists modified versions $\widetilde{\boldsymbol{z}}_{n}$ and $\widetilde{\boldsymbol{v}}_{n}$ of $\boldsymbol{z}_{n}$ and $\boldsymbol{v}_{n}$, respectively, such that they satisfy all the properties $(a)-(f)$ and additionally the following two properties

(g) $\alpha_{n} \widetilde{z}_{n} \rightarrow \mathbf{0}$, as $n \rightarrow \infty$ uniformly in $\boldsymbol{x} \in \Omega$;

(h) $\widetilde{z}_{n}(\boldsymbol{x})=\widetilde{\boldsymbol{v}}_{n}(\boldsymbol{x})=\boldsymbol{\psi}_{n}(\boldsymbol{x})=\mathbf{0}$ for all $\boldsymbol{x} \in \partial \Omega_{1}$.

Proof Step 1. Let $\boldsymbol{w}_{n}(\boldsymbol{x})=\left(w_{n}^{(1)}(\boldsymbol{x}), \ldots, w_{n}^{(m)}(\boldsymbol{x})\right)$ be defined by

$$
w_{n}^{(i)}(\boldsymbol{x})=\min \left\{z_{n}^{(i)}(\boldsymbol{x}),\left\|\boldsymbol{\psi}_{n}\right\|_{L^{\infty}\left(\Omega \backslash R_{n}\right)}\right\}, \quad i=1, \ldots, m .
$$

Then $\boldsymbol{w}_{n}(\boldsymbol{x})$ is Lipschitz continuous with $\left|\nabla \boldsymbol{w}_{n}(\boldsymbol{x})\right| \leq\left|\nabla \boldsymbol{z}_{n}(\boldsymbol{x})\right|$ for a.e. $\boldsymbol{x} \in \Omega$, and $\boldsymbol{w}_{n}(\boldsymbol{x})=$ $\boldsymbol{\psi}_{n}(\boldsymbol{x})$ for almost all $\boldsymbol{x} \in \Omega \backslash R_{n}$. Similarly, let $\boldsymbol{u}_{n}(\boldsymbol{x})=\left(u_{n}^{(1)}(\boldsymbol{x}), \ldots, u_{n}^{(m)}(\boldsymbol{x})\right)$ be defined by

$$
u_{n}^{(i)}(\boldsymbol{x})=\max \left\{w_{n}^{(i)}(\boldsymbol{x}),-\left\|\boldsymbol{\psi}_{n}\right\|_{L^{\infty}\left(\Omega \backslash R_{n}\right)}\right\}, \quad i=1, \ldots, m .
$$

Then $\boldsymbol{u}_{n}(\boldsymbol{x})$ is Lipschitz continuous with $\left|\nabla \boldsymbol{u}_{n}(\boldsymbol{x})\right| \leq\left|\nabla \boldsymbol{z}_{n}(\boldsymbol{x})\right|$ for a.e. $\boldsymbol{x} \in \Omega$, and $\boldsymbol{u}_{n}(\boldsymbol{x})=$ $\boldsymbol{\psi}_{n}(\boldsymbol{x})$ for almost all $\boldsymbol{x} \in \Omega \backslash R_{n}$. Therefore $\left|\nabla \boldsymbol{u}_{n}\right|^{2}$ is also equiintegrable and

$$
\alpha_{n}\left\|\boldsymbol{u}_{n}\right\|_{\infty} \leq \alpha_{n}\left\|\boldsymbol{\psi}_{n}\right\|_{\infty} \rightarrow 0, \text { as } n \rightarrow \infty .
$$

Step 2. Let $\psi_{0}$ be a $W^{1,2}$-weak limit of (a subsequence of) $\psi_{n}$. Then there exists a sequence $\widehat{\psi}_{n} \in C^{1}\left(\bar{\Omega} ; \mathbb{R}^{m}\right)$ such that $\widehat{\psi}_{n}$ vanishes on $\partial \Omega_{1}$, converges to $\psi_{0}$ in the $W^{1,2}$ norm and additionally satisfies

$$
\lim _{n \rightarrow \infty} \alpha_{n}\left\|\widehat{\psi}_{n}\right\|_{1, \infty}=0
$$

It follows from $\left|R_{n}\right| \rightarrow 0$, as $n \rightarrow \infty$ that $\boldsymbol{\psi}_{n}-\boldsymbol{u}_{n} \rightarrow \mathbf{0}$ weakly in $W^{1,2}$. Thus, by Rellich's lemma,

$$
\lim _{n \rightarrow \infty}\left\|\boldsymbol{u}_{n}-\widehat{\boldsymbol{\psi}}_{n}\right\|_{2}=0 .
$$

Let $\eta_{n}(\boldsymbol{x})$ be a Lipschitz cut-off function such $0 \leq \eta_{n}(\boldsymbol{x}) \leq 1$ and

$$
\eta_{n}(\boldsymbol{x})= \begin{cases}1, & \boldsymbol{x} \in \partial \Omega, \\ 0, & \operatorname{dist}(\boldsymbol{x}, \partial \Omega) \geq \delta_{n} .\end{cases}
$$

It is possible to do so, while ensuring that

$$
\left\|\nabla \eta_{n}\right\|_{\infty} \leq \frac{C}{\delta_{n}}
$$

where $C$ depends only on $\Omega$ and $\delta_{n} \rightarrow 0$, as $n \rightarrow \infty$ so slowly that

$$
\lim _{n \rightarrow \infty} \frac{\alpha_{n}\left\|\boldsymbol{u}_{n}\right\|_{\infty}}{\delta_{n}}=\lim _{n \rightarrow \infty} \frac{\alpha_{n}\left\|\widehat{\boldsymbol{\psi}}_{n}\right\|_{1, \infty}}{\delta_{n}}=\lim _{n \rightarrow \infty} \frac{\left\|\boldsymbol{u}_{n}-\widehat{\boldsymbol{\psi}}_{n}\right\|_{2}}{\delta_{n}}=0 .
$$

Step 3. Let

$$
\widetilde{\boldsymbol{z}}_{n}=\left(1-\eta_{n}\right) \boldsymbol{u}_{n}+\eta_{n} \widehat{\boldsymbol{\psi}}_{n} .
$$

It is obvious that $\widetilde{\boldsymbol{z}}_{n}(\boldsymbol{x})$ vanishes on $\partial \Omega_{1}$ and that $\alpha_{n} \widetilde{\boldsymbol{z}}_{n}(\boldsymbol{x}) \rightarrow 0$, as $n \rightarrow \infty$ uniformly in $\boldsymbol{x} \in \Omega$. Defining $\widetilde{\boldsymbol{v}}_{n}=\boldsymbol{\psi}_{n}-\widetilde{\boldsymbol{z}}_{n}$, proves (a), (g) and (h). By definition $\widetilde{\boldsymbol{z}}_{n}(\boldsymbol{x})=\boldsymbol{u}_{n}(\boldsymbol{x})$ for all 
$\boldsymbol{x}$, such that $\operatorname{dist}(\boldsymbol{x}, \partial \Omega) \geq \delta_{n}$. Hence, (b) and (e) are also established. The property (d) is a consequence of (b), (c) and (e). Let us now establish (f).

$$
\nabla \widetilde{\boldsymbol{z}}_{n}=\left(1-\eta_{n}\right) \nabla \boldsymbol{u}_{n}+\eta_{n} \nabla \widehat{\boldsymbol{\psi}}_{n}+\left(\widehat{\boldsymbol{\psi}}_{n}-\boldsymbol{u}_{n}\right) \otimes \nabla \eta_{n} .
$$

Hence,

$$
\alpha_{n}\left\|\nabla \widetilde{\boldsymbol{z}}_{n}\right\|_{\infty} \leq \alpha_{n}\left\|\nabla \boldsymbol{z}_{n}\right\|_{\infty}+\alpha_{n}\left\|\widehat{\boldsymbol{\psi}}_{n}\right\|_{1, \infty}+\frac{\alpha_{n}\left\|\widehat{\boldsymbol{\psi}}_{n}\right\|_{1, \infty}}{\delta_{n}}+\frac{\alpha_{n}\left\|\boldsymbol{u}_{n}\right\|_{\infty}}{\delta_{n}} .
$$

The property (f) is proved. To prove property (c), we observe that

$$
\left\|\nabla \widetilde{\boldsymbol{z}}_{n}-\nabla \boldsymbol{u}_{n}\right\|_{2} \leq\left\|\eta_{n}\left(\nabla \boldsymbol{u}_{n}-\nabla \widehat{\boldsymbol{\psi}}_{n}\right)\right\|_{2}+\frac{\left\|\boldsymbol{u}_{n}-\widehat{\boldsymbol{\psi}}_{n}\right\|_{2}}{\delta_{n}} \rightarrow 0, \text { as } n \rightarrow \infty
$$

because $\left|\nabla \boldsymbol{u}_{n}-\nabla \widehat{\boldsymbol{\psi}}_{n}\right|^{2}$ is equiintegrable and $\eta_{n}$ is bounded and supported on a set of small measure. Hence, (c) follows from the equiintegrability of $\left|\nabla \boldsymbol{u}_{n}\right|^{2}$.

Another correction has to be made to the statements of Theorems 5 and 6 . Theorem 5 has to apply to points in $\Omega \cup \partial \Omega_{1}$, while Theorem 6 to points in $\overline{\partial \Omega_{2}}$. Finally, no additional smoothness assumptions are required of $\partial \Omega_{1}$. We only need $\partial \Omega_{1}$ to be relatively open and coincide with the interior of its closure. If this condition is not satisfied we should just redefine $\partial \Omega_{1}$ as the interior of the closure of the original $\partial \Omega_{1}$. The set $\partial \Omega_{2}$ is then defined as $\partial \Omega \backslash \overline{\partial \Omega_{1}}$. 